\title{
A hybrid 3D SEM reconstruction method optimized for complex geologic material surfaces
}

\author{
Shang Yan, Aderonke Adegbule, Tohren C.G. Kibbey* \\ School of Civil Engineering and Environmental Science, University of Oklahoma, 202 W Boyd Street, Rm. 334, Norman, OK 73019, United States
}

\section{A R T I C L E I N F O}

\section{Keywords:}

SEM

3D Reconstruction

\begin{abstract}
A B S T R A C T
Reconstruction methods are widely used to extract three-dimensional information from scanning electron microscope (SEM) images. This paper presents a new hybrid reconstruction method that combines stereoscopic reconstruction with shape-from-shading calculations to generate highly-detailed elevation maps from SEM image pairs. The method makes use of an imaged glass sphere to determine the quantitative relationship between observed intensity and angles between the beam and surface normal, and the detector and surface normal. Two specific equations are derived to make use of image intensity information in creating the final elevation map. The equations are used together, one making use of intensities in the two images, the other making use of intensities within a single image. The method is specifically designed for SEM images captured with a single secondary electron detector, and is optimized to capture maximum detail from complex natural surfaces. The method is illustrated with a complex structured abrasive material, and a rough natural sand grain. Results show that the method is capable of capturing details such as angular surface features, varying surface roughness, and surface striations.
\end{abstract}

\section{Introduction}

The scanning electron microscope (SEM) is a useful tool to analyze the surface structures of natural and engineered materials (Bogner et al., 2007; Limandri et al., 2016; Rittscher et al., 2008). However, by itself, SEM can only provide two-dimensional images. Three dimensional surface reconstruction methods are required to obtain three dimensional information from two-dimensional SEM images. There are three major categories of three-dimensional reconstruction methods: single-view methods, multi-view methods, and hybrid methods (Tafti et al., 2015). Single-view methods, sometimes described as shape-fromshading methods, calculate three-dimensional information based on one or more images taken from the same view point. Photometric stereo, where images with different lighting or sensor configurations are used to calculate surface elevations, is one example of a single-view method. The method and its variations have been widely used, and are particularly well suited to extracting three-dimensional information from smooth surfaces (Argyriou and Petrou, 2008; Drzazga et al., 2005; Marinello et al., 2008; Paluszyński and Slowko, 2005; Pintus et al., 2008; Slowko and Krysztof, 2013; Warren, 2007; Woodham, 1980). Multi-view methods make use of two or more images taken from different viewpoints to reconstruct surfaces based on the mathematics of projection. Stereo matching is the most common multi-view approach (Minnich et al., 1999; Samak et al., 2007; Stampfl et al., 1996; Zisserman, 2004). The method is based on finding matching points on a surface in stereo images captured at different angles, and then calculating the elevation of the point in three dimensions based on the coordinates in the two-dimensional images. One of the major challenges of the method is accurate matching of points between the images, because a single mismatched point will skew the final threedimensional surface; approaches from neural adaptive learning or machine learning have been used with success to improve matching (Binaghi et al., 2004; Tafti et al., 2016). Unlike single-view methods, stereo matching methods are best suited to images with hard edges or other distinct features to allow accurate identification of matching points between images. However, stereo-matching methods are not able to accurately determine three dimensional information for portions of surfaces between the identified matching points, so interpolation must be used to estimate the surface shape in those regions. Furthermore, even with sub-pixel matching point identification, pixel resolution tends to limit the accuracy of three dimensional information determined from matching point methods, because small differences in position between two images often correspond to large steps in elevation. Hybrid methods combine aspects of both single-view and multi-view methods, using multi-view methods to determine the major three-dimensional structure, and then single-view methods to fill in

\footnotetext{
* Corresponding author.

E-mail address: kibbey@ou.edu (T.C.G. Kibbey).
} 
detail (e.g., (Beil and Carlsen, 1991; Danzl and Scherer, 2002)).

The work described in this paper centers around a hybrid method optimized to extract maximum detail from complex natural surfaces. The method uses a multi-scale windowing method to reject mismatched points from stereoscopic images, and then a novel shape-from-shading approach that incorporates the detector location and response into the reconstruction algorithm, and incorporates intensity information both from two separate images and within a single image. The method is specifically designed for images captured using a single in-chamber secondary electron detector.

\section{Methodology}

\subsection{SEM imaging}

Images for the work were captured with a Zeiss NEON 40 EsB highresolution scanning electron microscope. Images were captured at a resolution of $1024 \times 768$, with pixel sizes ranging from 1.002 to $1.787 \mu \mathrm{m}$. All materials were sputtered with a gold-palladium alloy prior to imaging. Images were captured using the in-chamber secondary electron (SE) detector at a voltage of $5.0 \mathrm{kV}$. For images used for three dimensional reconstruction, stereo image pairs were taken the sample stage tilted $6^{\circ}$ apart $\left( \pm 3^{\circ}\right.$ from the horizontal).

\subsection{Description of the hybrid method}

The hybrid method described in this paper consists of a stereo matching method which is used to determine the coarse three dimensional structure of the surface, and then a shape-from-shading algorithm to extract additional fine detail from the SEM images. The subsequent sections describe each of these two components of the hybrid method.

\subsubsection{Stereo matching component}

The stereo matching component of this work is based around the geometry analysis method described by Piazzesi (1973). The equations calculate the elevation of a surface point based on the geometry of projection:

$x \approx \frac{1}{M} \frac{X_{1}+X_{2}}{2 \cos \Delta \psi} \quad y \approx \frac{Y_{1}}{M}=\frac{Y_{2}}{M} \quad \mathrm{Z} \approx \frac{1}{M} \frac{X_{1}+X_{2}}{2 \sin \Delta \psi}$

where $x, y$ and $z$ are coordinates in three-dimensional space, $M$ is the image magnification, and $\left(X_{1}, Y_{1}\right)$ and $\left(X_{2}, Y_{2}\right)$ are two-dimensional pixel coordinates for the same point in space in Images 1 and 2, respectively, for two images taken of an object tilted at angles of $\pm \Delta \psi$ about the $y$ axis. The equations are valid for small angles, and for systems where the distance from the imaging plane to the object is much greater than the size of the object.

The major challenge in any stereo matching method is the accurate identification of coordinates of matching points between the two images. A number of different methods have been used with success in the past (Binaghi et al., 2004; Eastman and Waxman, 1987; Hoff and Ahuja, 1989; Kanade and Okutomi, 1994; Pollard et al., 1985). For this work, candidate matching points are selected using the following approach: Edges and extreme light or dark points are identified in one stereo image by taking the difference of images smoothed to different degrees (Lowe, 2004). For this work, Gaussian-Laplace filters were used, with $\sigma$ values of 1.8 and 2.0 for the two smoothed images. Candidate points were identified as all positive local maxima or negative local minima extreme points along all $x$ scan lines. Matching points for these were then identified in the second stereo image by first creating a transformed version of each stereo image containing the absolute value of the first derivative of intensity with respect to $x$ $(|\partial I / \partial x|)$. The use of absolute value first derivative images was found to slightly improve performance in preliminary tests with repetitive synthetic images. Matches were identified by searching for areas on each transformed image with the highest correlation coefficients. Four search window sizes were used: $33 \times 33$ pixels, $17 \times 17$ pixels, $9 \times 9$ pixels, and $5 \times 5$ pixels. The coordinate of the matching point was taken as the center of the search window, and the maximum was identified with subpixel accuracy by fitting a Centripetal Catmull-Rom spline to the cross-correlation values surrounding the apparent maximum (Barry and Goldman, 1988). Matching points with cross-correlation coefficients less than 0.7 were eliminated, as were points where the maximum difference between the matching point position for the four window sizes exceeded 0.8 pixels. This approach and the selected thresholds yielded a set of accurate matching points, with few apparent artifacts. A final filter was applied to remove any adjacent identified matching points where the calculated three dimensional slope between adjacent points $(\partial z / \partial x$, as determined from preliminary elevations determined using Eq. (1)) was greater than 60 pixels per pixel; this typically removed a number of matching points in the low single digits. Final stereo-matching elevation maps were created by applying Eq. (1) to all match points, interpolating to a uniform grid by creating a polygonal mesh connecting the points, and then identifying new points at uniform spatial intervals by linearly interpolating within the polygons. Because there are no matching points directly along the elevation map edges (because of the widths of the search windows), the outer edges of the interpolated map are trimmed to remove surface points extrapolated beyond the known data. The resulting elevation maps were used as inputs to the shape-from-shading component of the hybrid method described in the next section. Note that all thresholds and constants indicated in the section above were selected empirically, and were found to work well for the conditions tested; however, other imaging systems or materials might necessitate different values. Note also that because the stereo matching component of the work is not intended as a final product, but rather is used to provide a coarse preliminary elevation map as the input to the shape-from-shading component of the hybrid method, a number of different stereo matching methods would likely work equally well for this purpose.

\subsubsection{Shape from shading component}

The shape-from-shading component of the hybrid method starts with the stereoscopic elevation map from the previous section, and then uses intensity information in the two images to refine the surface to capture the effect of detail in the images. It has long been recognized that elevation data can be extracted from the intensity of SEM image pixels by relating intensity to surface slope. In particular, a number of authors have used equations based on the intensity values at a point in three dimensional space identified in two images where the sample was rotated through a small angle $\pm \Delta \psi$ (e.g., (Beil and Carlsen, 1991; Kholodilov et al., 1987; Newbury, 1975)). The equations derived in these studies generally consider the detector intensity to be proportional to sec $\alpha$, where $\alpha$ is the angle between the surface normal and the incident electron beam. The reason for this functional form is that the secondary electron yield of a surface is proportional to sec $\alpha$ (Newbury, 1975; Reimer, 1998). However, it is important to recognize that this functional form does not take into account the fact that only a portion of secondary electrons will be captured by the detector. Specifically, only secondary electrons that leave the surface at an angle within $90^{\circ}$ of the vector between the detector and the sample are captured by the detector. This gives detected intensities proportional to $\sec \alpha(1+\cos \beta)$, where $\beta$ is the angle between the detector and the sample, and also creates the appearance of shading from one side of an object. (See the accompanying Supplementary Material section for additional discussion and a derivation of this functional form.)

The equations developed in this work are based on the relationship in Eq. (2):

$I=k \cdot \sec \alpha(1+\cos \beta)+c$

where $I$ is the measured intensity at some point $(x, y)$, and $k$ and $c$ are constants for a specific set of imaging parameters. Note that the 


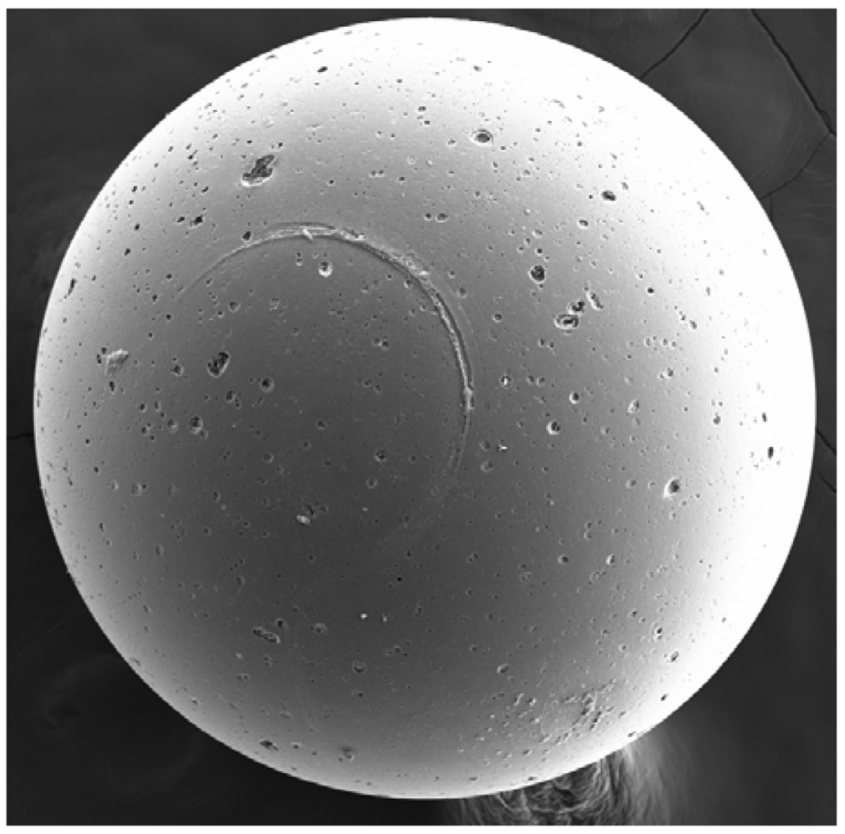

Fig. 1. Image of a $685 \mu \mathrm{m}$ diameter glass sphere used to characterize detector response and direction.

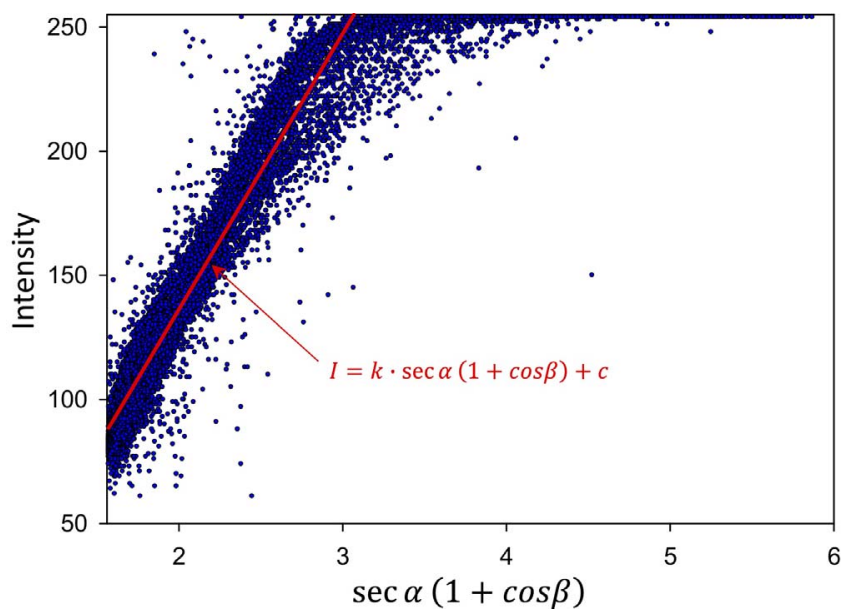

Fig. 2. Relationship between intensity and angles $\alpha$ and $\beta$ determined from the sphere in Fig. 1. Data points along top edge correspond to points where the detector is saturated (upper right of the sphere in Fig. 1).

constant $c$ is included to account for the fact that images captured are not raw signals, but rather are processed for display by adjusting contrast and brightness. The constants in Eq. (2) can be determined by processing an image of a sphere collected with the same imaging parameters used for other surfaces. For example, Fig. 1 shows the image of a $685 \mu \mathrm{m}$ glass sphere (SI-BG05, Scientific Industries (Bohemia, NY)) used for this purpose. Because the surface normal can be calculated at all points on the sphere, it is possible to relate the angles $\alpha$ and $\beta$ to intensity. Fig. 2 shows a plot of image intensities from Fig. 1 vs. $\sec \alpha(1+\cos \beta)$, where nonlinear optimization was used to determine the constants $k$ and $c$, as well as the components of the detector position vector, $\boldsymbol{d}$, a unit vector from the object to the detector $(\cos \beta=\boldsymbol{n} \cdot \boldsymbol{d}$, where $\boldsymbol{n}$ is the unit normal vector to the surface at point $(x, y))$. The beam source is assumed to be positioned at direction $(0,0,1)$ from the surface, so $\sec \alpha=(\boldsymbol{n} \cdot(0,0,1))^{-1}$. Note that edge detection was used to exclude points associated with surface defects on the sphere in Fig. 1 in the calculations. Note also that the image in Fig. 1 is slightly overexposed, a fact that results in the points in Fig. 2 that have the maximum intensity (254) but do not follow the linear trend. (In Fig. 1, these points are on the upper right of the sphere.) The non-saturated data in Fig. 2 correspond to the constants $k=111.135 \pm 0.3794, c=-$ $85.965 \pm 0.1945$, and $\boldsymbol{d}=(0.418,-0.458,0.785)$, i.e., the detector is located up and to the right in images (the $y$ axis is positive downward). The angle described by the vector $\boldsymbol{d}$ has a one-standard deviation error of $0.11^{\circ}$, as determined from the nonlinear optimization least squares fit.

The information in Eq. (2) is used to develop two equations relating intensity to surface morphology. The first, Eq. (3), uses the intensity values of a single point in space in two images of an object that have been rotated about the $y$-axis by a small angle $\pm \Delta \psi$.

$\tan \psi=\frac{\partial z}{\partial x}=\frac{\left(I_{2}-I_{1}\right)-2 d_{x} k \tan \Delta \psi}{\left(I_{2}+I_{1}-2 c-2 d_{z} k\right) \tan \Delta \psi}$

where $I_{1}$ is the intensity of a specific point in space in an image where the object has been rotated at a small angle $-\Delta \psi$ about the $y$-axis, and $I_{2}$ is the intensity of a specific point in space in an image where the object has been rotated at a small angle $+\Delta \psi$, about the $y$-axis, $d_{x}$ and $d_{z}$ are the $x$ and $z$ components of the vector $\boldsymbol{d}$, respectively, and $c$ and $k$ are constants from Eq. (2). Note that the derivation of Eq. (3) follows a similar approach to that described by Kholodilov et al. (1987), but has been derived to include the location of the secondary electron detector. (A detailed derivation of Eq. (3) is provided in the accompanying Supplementary Material section.) It should be emphasized that $I_{1}$ and $I_{2}$ in Eq. (3) do not correspond to the same pixel coordinates in each image, but rather correspond to the same point in three dimensional space. Eq. (3) is used to relate image coordinates to space coordinates. The pixel locations of $I_{1}$ and $I_{2}$ are initially based on the interpolated surface from the stereo matching component, and are updated as the surface is progressively refined using the shape-from-shading information.

Because Eq. (3) only describes slopes in the $x$-direction, additional information is needed to maintain continuity between adjacent $x$ scanlines. Others (e.g., (Danzl and Scherer, 2002)) have used minimization of the second derivatives perpendicular to scanlines for this purpose. However, this approach can yield an artificially smoothed surface, blurring any sharp transitions in the final surface. Because the objective of this work was to resolve as much detail as possible from the surface, Eq. (4) was developed to relate intensity differences in the $y$ direction in a single image and calculated slopes in the $x$-direction to the second derivative:

$\left.\frac{\partial^{2} z}{\partial y^{2}}\right|_{I} \approx \frac{1}{2 \cdot d_{y}}\left(\frac{I_{i, j+1}-I_{i, j-1}}{k}-\left(\tan \psi_{i, j+1}-\tan \psi_{i, j-1}\right)-\left(\frac{1}{n_{z i, j+1}}-\frac{1}{n_{z i, j-1}}\right)\right)$

where $d_{y}$ is the $y$ component of the vector $\boldsymbol{d}, \tan \psi$ is angle of the surface from the x-axis $(\partial z / \partial x)$ from Eq. (3), and $n_{z}$ is the $z$-component of the surface normal, evaluated iteratively from the calculated surface as it converges. The indices $i$ and $j$ in Eq. (4) correspond to points in space, not on the image; $i$ corresponds to the $x$-direction, while $j$ corresponds to the $y$-direction. Values of $I$ are taken from one image, with their pixel coordinates calculated as before through the use of Eq. (1). Values of $\tan \psi$ are taken from those determined in Eq. (3), and values of $n_{z}$ are iteratively updated from the calculated surface elevation map. (See the accompanying Supplementary Material section for a detailed derivation of Eq. (4).)

It should be noted that because secondary electron yield can vary with material properties, the use of any shape-from-shading calculations such as those described in Eqs. (3) and (4) could potentially be complicated by the presence of heterogeneous material surfaces. For the method described here, Eq. (3) is relatively insensitive to heterogeneity because it compares the same points in space on two different images. However, Eq. (4) could misidentify spatial material differences as second derivative changes, causing convergence problems. The use of a sputtered gold coating and imaging at low voltages (e.g., $5 \mathrm{kV}$ or 

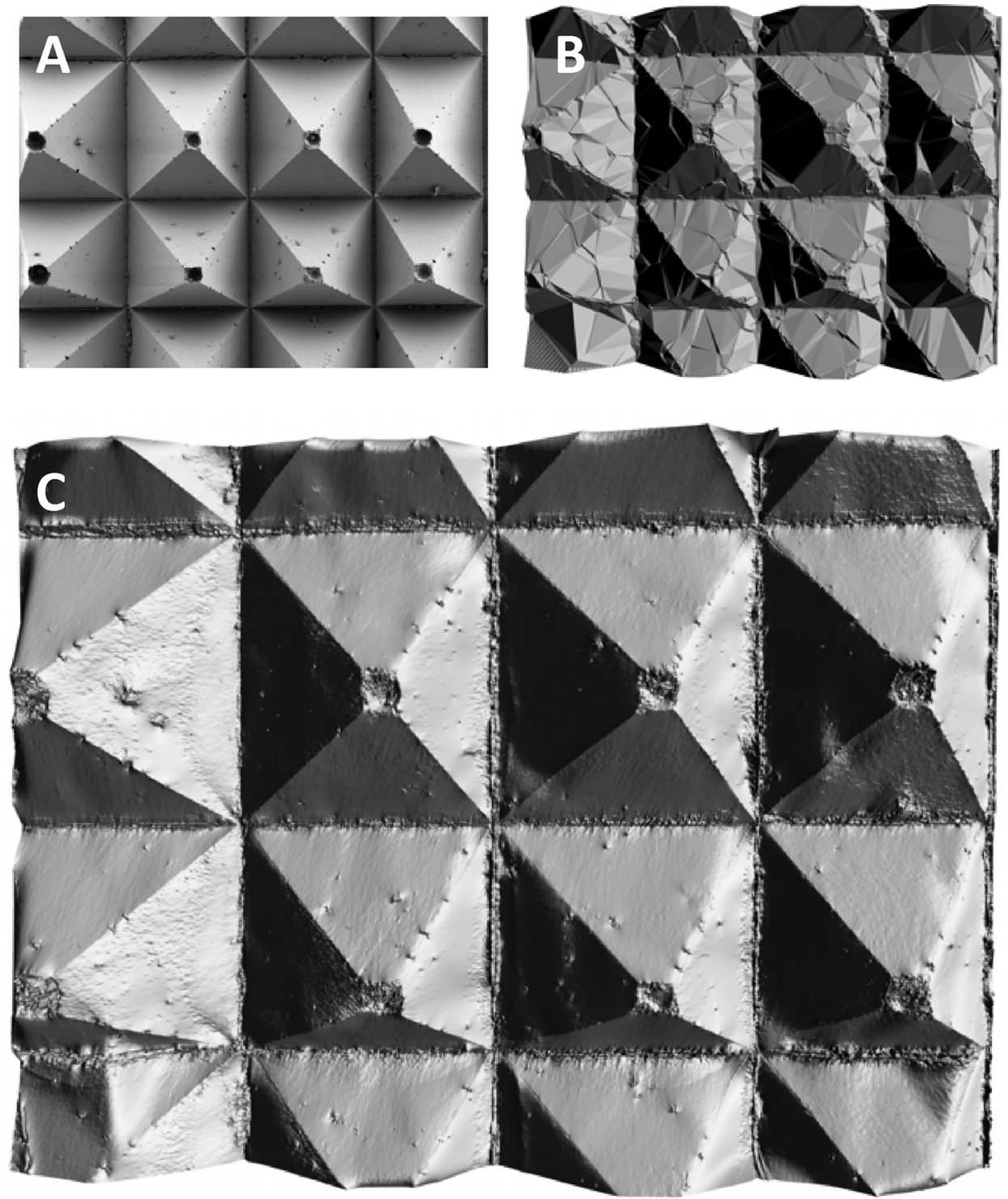

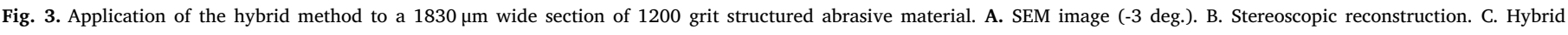
reconstruction.

less) can largely mitigate this problem, reducing material contrast differences (Reimer, 1998). However, like most shape-from-shading based methods, the method is most applicable to single-material surfaces. To calculate the surface elevation map, an iterative approach is applied. Like the approach described by Beil and Carlsen (1991) and Danzl and Scherer (2002), a cost function (Eq. (5)) is progressively minimized to determine the hybrid method surface elevation map:

$C=\sum_{(i, j)}\left(w_{\psi} C_{\psi}+w_{\phi} C_{\phi}\right)$

where $w_{\psi}$ and $w_{\phi}$ are weighting coefficients for the two components of the cost function, $C_{\psi}$ and $C_{\phi}$, as defined in Eqs. (6) and (7):

$C_{\psi}=\left(\left(z_{i, j}-z_{i-1, j}\right)-\tan \psi_{i, j}\right)^{2}$

$C_{\phi}=\left(\left(z_{i, j+1}-2 z_{i, j}+z_{i, j-1}\right)-\left.\frac{\partial^{2} z}{\partial y^{2}}\right|_{I}\right)^{2}$

where $\tan \psi_{i, j}$ is from Eq. (3), and $\left.\frac{\partial^{2} z}{\partial y^{2}}\right|_{I}$ is from Eq. (4). For this work, the weighting coefficients in Eq. (5) are both set equal to 1.0. Note that while the stereo matching surface is used as the initial elevation map for the shape-from-shading calculations, it is not included in the cost function for optimization. The reason for this is that inclusion of the stereo matching surface in the cost function would blur the resulting final elevation map, because stereo matching surfaces are partially interpolated. Optimization of the surface is conducted using the Progressive Optimality Algorithm (POA) (Howson and Sancho, 1975) to minimize Eq. (5) by adjusting elevations throughout the elevation map to progressively reduce local $C$ values. Multiple iterations are conducted until the surface converges; on the order of 100 iterations were required for the surfaces explored in this paper.

\section{Results and discussion}

Fig. 3 shows use of the method on a commercial structured abrasive material, a portion of a 1200-grit $3 \mathrm{M}$ Trizact sanding disk, model 237AA. The material was selected because of its distinct, sharp edges and flat planes, but also because of the small attached particulates and other interesting surface defects. Some reconstruction methods struggle to reconstruct objects with sharp edges, yielding rounded elevation maps. Furthermore, objects with repeating parallel lines can be challenging from the standpoint of stereo identification, because of 

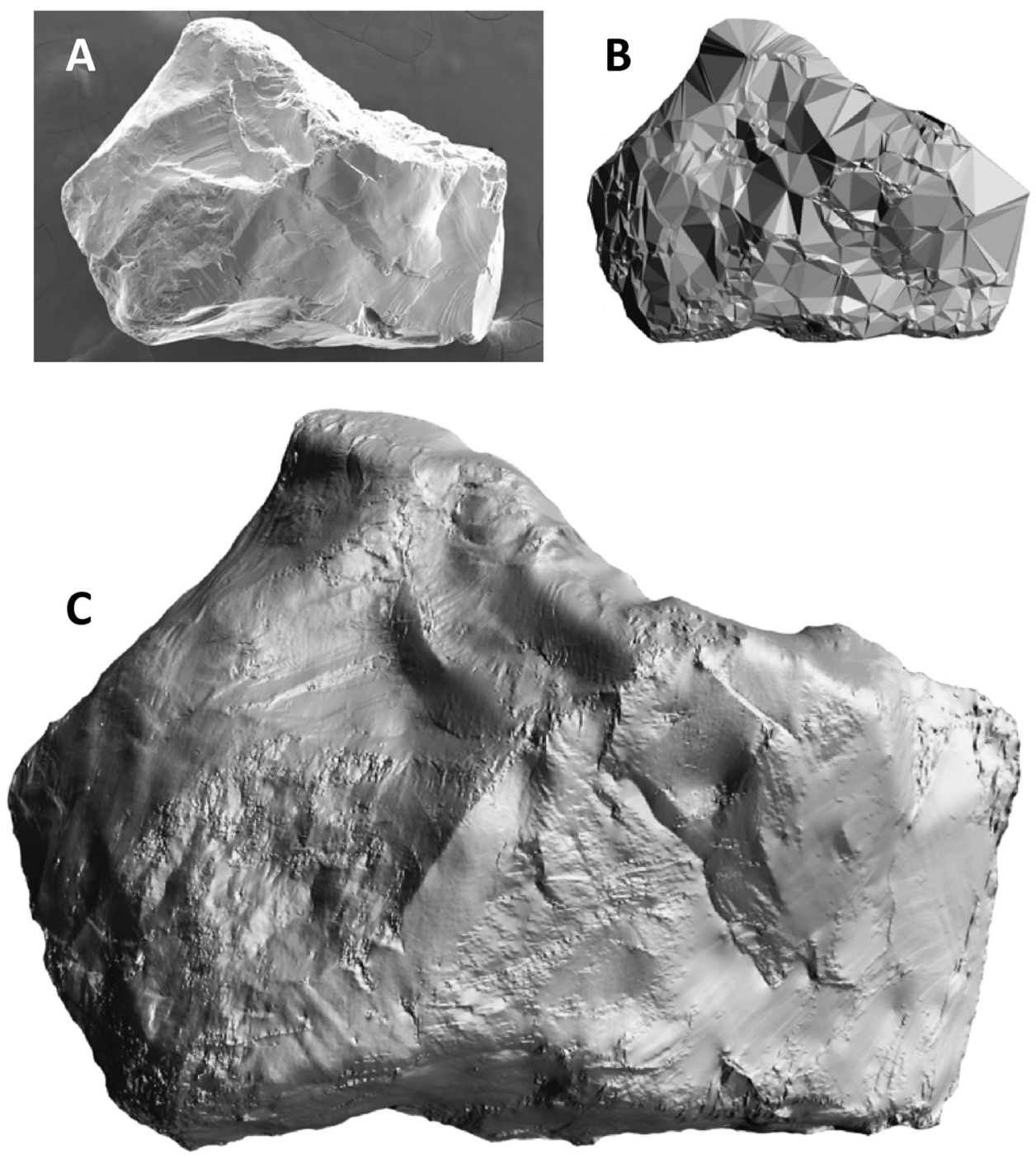

Fig. 4. Application of the hybrid method to a $1000 \mu \mathrm{m}$ wide grain of beach sand. A. SEM image (-3 deg.). B. Stereoscopic reconstruction. C. Hybrid reconstruction.

the difficulties associated with unique identification of matching points between images.Fig. 3A shows one of the two SEM images used for the reconstruction (because of the similarity between the images, only one is included here; See the accompanying Supplementary Material section for side-by-side presentation of all stereo images.) Fig. 3B shows the stereo reconstruction of the surface, and Fig. $3 \mathrm{C}$ shows the final hybrid reconstruction. Note that reconstructions in the paper are rendered using the TVTK framework as they would appear with visual light illumination. Although a directional light source has been positioned to roughly approximate the detector position (upper right in the images), the physics of illumination differs from that of secondary electron detection, so shaded faces appear in the reconstruction renderings (Fig. 3B, C) where they might otherwise be bright in an SEM image (Fig. 3A) as a result of the sec $\alpha$ in Eq. (2) (e.g., steep faces with surface normal vectors pointing to the left). Nevertheless, it is apparent from the figure that the hybrid reconstruction method does a very good job of capturing the detail in the SEM image, including the sharp edges and angular facets, the attached dust particles, and the small indentations at the top of individual pyramids. Because the hybrid method starts with the stereo elevation map, any errors in the stereo reconstruction can produce artifacts in the final hybrid reconstruction. For example, the lower left corner of Fig. 3B shows some apparent artifacts (not uncommon on image boundaries). The artifact propagates into the hybrid reconstruction in Fig. 3C, appearing as warping in the lower left corner of the image. Note also that although the hybrid method does a very good job of reproducing the dust particles on the surface, the fact that the reconstructed elevation map is in the form $z=f(x, y)$ means that they are not identified as separate objects, so do not appear as distinct as in the original images. Finally, as is the case with many shape-from-shading methods, shadows have the potential to confuse the reconstruction process, because they show pixel intensities that are not directly related to surface slope. While the optimization method used here reduces this effect (in particular, Eq. (7)), some of the minor surface warping around the attached dust particles in Fig. 3C likely results in part from this effect. Fig. 4 shows use of the method to reconstruct the surface of a $1000 \mu \mathrm{m}$ wide beach sand grain collected from Sandwich, Massachusetts. Fig. 4A shows one of the two stereo images used in the reconstruction. (See the accompanying Supplementary Material section for the image pair.) Fig. 4B and C show the stereo and hybrid reconstructions of the surface. Note that the background has been removed from both reconstructions to allow easier comparison with the SEM image. (Rendered reconstructions including the background are included in the accompanying Supplementary Material section.) Overall, the hybrid reconstruction method does a very good job of duplicating much of the surface detail of the grain, although it is apparent that the reconstruction is somewhat less angular than the original SEM image; in particular, some of the steepest faces and their adjacent edges are rounded in Fig. 4C where they are clearly angular in the original image. Areas where the method works well include in its ability to duplicate the distinct surface striations throughout the grain, 
as well as patches of locally-varying roughness. The level of detail with which these features can be resolved should be sufficient for many uses of grain surface elevation maps, including detailed simulations of fluid configuration and flow on surfaces among other applications (e.g. (Kibbey, 2013).

\section{Conclusions}

This paper presents a new hybrid three-dimensional reconstruction method that is optimized to extract maximum detail from SEM images of natural materials. The underlying equations for the method are derived for use with a single in-chamber secondary electron detector, to take advantage of the additional directional information available from the detector response. The method makes use of an imaged sphere to calculate both detector location and image response, and then a two stage reconstruction method to create an elevation map. The resulting elevation maps are able to reproduce angular features in the original SEM images, including surface striations and patches of locally-varying roughness, making the method well-suited to applications that require extraction of detail from SEM images of natural materials. Limitations of the method are those that challenge most shape-from-shading-based methods, including surface warping caused by shadows, and limitations in the ability to represent overlapping objects with a single-valued $(z=f(x, y))$ elevation map.

\section{Acknowledgments}

This material is based upon work supported by the National Science Foundation under Grant No. 1446264. The authors thank Dr. Preston Larson of the University of Oklahoma Samuel Roberts Noble Electron Microscopy Laboratory for assistance with the imaging conducted for this work.

\section{References}

Argyriou, V., Petrou, u.M., 2008. Recursive photometric stereo when multiple shadows and highlights are present, Computer Vision and Pattern Recognition, 2008, CVPR 2008. IEEE Conference On IEEE 1-6.

Barry, P.J., Goldman, R.N., 1988. A recursive evaluation algorithm for a class of CatmullRom splines. ACM SIGGRAPH Comput. Graphics 22, 199-204.

Beil, W., Carlsen, I., 1991. Surface reconstruction from stereoscopy and shape from shading in SEM images. Mach. Vision. Appl. 4, 271-285.

Binaghi, E., Gallo, I., Marino, G., Raspanti, M., 2004. Neural adaptive stereo matching. Pattern Recogn. Lett. 25, 1743-1758.

Bogner, A., Jouneau, P.H., Thollet, G., Basset, D., Gauthier, C., 2007. A history of scanning electron microscopy developments: towards wet-STEM imaging. Micron 38,
$390-401$.

Danzl, R., Scherer, S., 2002. Integrating Shape from Shading and Shape from Stereo for Variable Reflectance Surface Reconstruction from SEM Images. Proceedings of 26th Workshop of the Austrian Association for Pattern Recognition (AAPR) 281-288.

Drzazga, W., Paluszynski, J., Slowko, W., 2005. Three-dimensional characterization of microstructures in a SEM. Meas. Sci. Technol. 17, 28.

Eastman, R.D., Waxman, A.M., 1987. Using disparity functionals for stereo correspondence and surface reconstruction. Comput. Vision Graphics 39, 73-101.

Hoff, W., Ahuja, N., 1989. Surfaces from stereo: integrating feature matching, disparity estimation, and contour detection. IEEE Trans. Pattern Anal. Mach. Intell. 11, 121-136.

Howson, H., Sancho, N., 1975. A new algorithm for the solution of multi-state dynamic programming problems. Math. Program. 8, 104-116.

Kanade, T., Okutomi, M., 1994. A stereo matching algorithm with an adaptive window theory and experiment. IEEE Trans. Pattern Anal. Mach. Intell. 16, 920-932.

Kholodilov, O.V., Grigoryev, A.Y., Myshkin, N.K., 1987. Reconstruction of true topographies of solid-surfaces in scanning electron-microscopes using secondary electrons. Scanning 9, 156-161.

Kibbey, T.C.G., 2013. The configuration of water on rough natural surfaces: implications for understanding air-water interfacial area, film thickness, and imaging resolution. Water Resour. Res. 49, 4765-4774.

Limandri, S., Josa, V.G., Valentinuzzi, M.C., Chena, M.E., Castellano, G., 2016. 3D scanning electron microscopy applied to surface characterization of fluorosed dental enamel. Micron 84, 54-60.

Lowe, D.G., 2004. Distinctive image features from scale-invariant keypoints. Int. J. Comput. Vision 60, 91-110.

Marinello, F., Bariani, P., Savio, E., Horsewell, A., De Chiffre, L., 2008. Critical factors in SEM 3D stereo microscopy. Meas. Sci. Technol. 19, 065705.

Minnich, B., Leeb, H., Bernroider, E., Lametschwandtner, A., 1999. Three-dimensional morphometry in scanning electron microscopy: a technique for accurate dimensional and angular measurements of microstructures using stereopaired digitized images and digital image analysis. J. Microsc. 195, 23-33.

Newbury, D.E., 1975. Image formation in the scanning electron microscope. In: Goldstein, J., Yakowitz, H. (Eds.), Practical Scanning Electron Microscopy: Electron and Ion Microprobe Analysis. Plenum Press, New York pp. xviii, 582 p.

Paluszyński, J., Slowko, W., 2005. Surface reconstruction with the photometric method in SEM. Vacuum 78, 533-537.

Piazzesi, G., 1973. Photogrammetry with the scanning electron microscope. J. Phys. E: Sci. Instrum. 6, 392.

Pintus, R., Podda, S., Vanzi, M., 2008. An automatic alignment procedure for a foursource photometric stereo technique applied to scanning electron microscopy. IEEE Trans. Instrum. Meas. 57, 989-996.

Pollard, S.B., Mayhew, J.E.W., Frisby, J.P., 1985. Pmf - a stereo correspondence algorithm using a disparity gradient limit. Perception 14, 449-470.

Reimer, L., 1998. Scanning Electron Microscopy: Physics of Image Formation and Microanalysis, 2nd ed. Springer, Berlin; New York.

Rittscher, J., Machiraju, R., Wong, S.T., 2008. Microscopic Image Analysis for Life Science Applications. Artech House.

Samak, D., Fischer, A., Rittel, D., 2007. 3D reconstruction and visualization of microstructure surfaces from 2D images. CIRP Ann. Manuf. Technol. 56, 149-152.

Slowko, W., Krysztof, M., 2013. Detector system for three-Dimensional imaging in the variable Pressure/Environmental SEM. Acta Phys. Pol. A 123, 877-879.

Stampfl, J., Scherer, S., Gruber, M., Kolednik, O., 1996. Reconstruction of surface topographies by scanning electron microscopy for application in fracture research. Appl. Phys. A Mater. 63, 341-346.

Tafti, A.P., Kirkpatrick, A.B., Alavi, Z., Owen, H.A., Yu, Z., 2015. Recent advances in 3D SEM surface reconstruction. Micron 78, 54-66.

Tafti, A.P., Holz, J.D., Baghaie, A., Owen, H.A., He, M.M., Yu, Z., 2016. 3DSEM + + : adaptive and intelligent 3D SEM surface reconstruction. Micron 87, 33-45.

Warren, J.S., 2007. Modern Optical Engineering. The Design of Optical Systems .

Woodham, R.J., 1980. Photometric method for determining surface orientation from multiple images. Opt. Eng. 19 (191139-191139).

Zisserman, R.H.A., 2004. Multiple View Geometry in Computer Vision . 